\title{
Geometrical properties of intelligent spin states and time evolution of coherent states
}

\author{
R Arvieu胴 and P Rozmej $\ddagger \S$ \\ $\dagger$ Institut des Sciences Nucléaires, F-38026 Grenoble, France \\ $\ddagger$ Instytut Fizyki, Uniwersytet MCS, 20-031 Lublin, Poland \\ $\S$ Gesellschaft für Schwerionenforschung, D-64220 Darmstadt, Germany
}

\begin{abstract}
We remind the properties of the intelligent (and quasi-intelligent) spin states introduced by Aragone et al. We use these states to construct families of coherent wave packets on the sphere and we sketch the time evolution of these wave packets for a rigid body molecule.

PACS numbers: $03.65 . \mathrm{Sq}$
\end{abstract}

Submitted to: Institute of Physics Publishing

\section{Introduction}

The eigenstates of the square of the angular momentum operator $L^{2}$ which are also eigenstates of $L_{x}+i \eta L_{y}$, where $\eta$ is a real parameter, have been called the intelligent spin states [1] and have been the subject of intensive analytical studies [2, 3]. However, at least to our knowledge, one does not find in the literature any discussion of their geometrical interpretation and of their utility in the construction of angular momentum coherent states. In completing a recent work [4] on the time evolution of coherent states built from a subclass of those states we came to conclusion that such a discussion had still to be presented.

In this short article we will first show in section 2 that the parameter $\eta$ enables to define squeezed states on the sphere i.e. states for which the uncertainties $\Delta L_{x}^{2}$ and $\Delta L_{y}^{2}$ can be varied at will. It is however for real values of $\eta$ that the product of the uncertainties has a minimum value. In section 3 we will show that the states can be classified into three categories, a result already found in ref. [3], and that the well known Radcliffe's states belong to one of these categories. In section t we will study the angular localization and the partial wave expansion of a particular class generated from an exponential coherent state on the sphere. This state was introduced by us elsewhere [4]. Finally in section 5 we will study the time evolution of this particular set

|| E-mail: arvieu@in2p3.fr and rozmej@tytan.umcs.lublin.pl 
of states in the same line as in [4] i.e. assuming that the wave packets evolve with the hamiltonian of a rigid body with moment of inertia $J$ i.e.

$$
H=\frac{\hbar^{2}}{2 J} L^{2} .
$$

In particular we will show that the scenario of fractional revivals found in 8 is well exhibited by the family of wave packets studied in section 4 .

\section{Squeezed states on the sphere}

Let us enumerate a few general properties of the states $|w, \eta\rangle$ which are eigenstates of $L_{x}+i \eta L_{y}$ with a complex value of $\eta$. In this first part there is no need to assume that they are eigenstates of $L^{2}$. These states are the normalized states which obey the equation:

$$
\left(L_{x}+i \eta L_{y}\right)|w, \eta\rangle=w|w, \eta\rangle \text {. }
$$

It is a simple exercise, already discussed in references [5] and [6], to prove equations (3-7). First of all

$$
|\eta|^{2}=\frac{\Delta L_{x}^{2}}{\Delta L_{y}^{2}}
$$

i.e. $|\eta|$ can be called the squeezing parameter (with the definitions $\Delta L_{i}^{2}=\left\langle L_{i}^{2}\right\rangle-$ $\left.\left\langle L_{i}\right\rangle^{2}, i=x, y\right)$. The phase $\alpha$ of $\eta$ determines the ratio of the average value of the anticommutator of $L_{x}$ with $L_{y}$ to the average of their commutator since

$$
\tan \alpha=\frac{\left\langle\left\{L_{x}, L_{y}\right\}\right\rangle-\left\langle L_{x}\right\rangle\left\langle L_{y}\right\rangle}{\left\langle L_{z}\right\rangle} .
$$

Finally the product of the uncertainties is given by:

$$
\Delta L_{x}^{2} \Delta L_{y}^{2}=\frac{1}{4}\left[\left\langle L_{z}\right\rangle^{2}+\left|\left\langle\left\{L_{x}, L_{y}\right\}\right\rangle-\left\langle L_{x}\right\rangle\left\langle L_{y}\right\rangle\right|^{2}\right]=\frac{1}{4} \frac{\left\langle L_{z}\right\rangle^{2}}{\cos ^{2} \alpha} .
$$

The average values of $L_{x}$ and $L_{y}$ are fixed both by the parameter $\eta$ and the eigenvalue $w$ by:

$$
\left\langle L_{x}\right\rangle=\frac{\eta w^{*}+w \eta^{*}}{\eta+\eta^{*}}, \quad\left\langle L_{y}\right\rangle=\frac{1}{i} \frac{w-w^{*}}{\eta+\eta^{*}} .
$$

The eigenstates corresponding to a real parameter $\eta$ satisfy the important minimum uncertainty relation from which the work [1] was initiated

$$
\Delta L_{x}^{2} \Delta L_{y}^{2}=\frac{1}{4}\left\langle L_{z}\right\rangle^{2}
$$




\section{Classification of the squeezed states}

The eigenvalue $w$ can be obtained very simply in the basis of eigenstates of $L^{2}$ with eigenvalue $l(l+1)$ if one uses the observation [3] that, within a constant factor $\sqrt{1-\eta^{2}}$, the operator $L_{x}+i \eta L_{y}$ is one of the three generators of an $\mathrm{SU}(2)$ algebra. The set of operators satisfying this algebra is defined as

$$
\mathcal{L}_{3}=\frac{L_{x}+i \eta L_{y}}{\sqrt{1-\eta^{2}}}, \quad \mathcal{L}_{ \pm}= \pm\left(\frac{\eta L_{x}+i L_{y}}{\sqrt{1-\eta^{2}}}\right)-L_{z} .
$$

Therefore there are $2 l+1$ solutions to equation (2); instead of $w$ one uses simply the eigenvalue of $\mathcal{L}_{3}$ with $k=-l, \ldots,+l$ and the formula

$$
w=k \sqrt{1-\eta^{2}} .
$$

Let us denote by $|l, k, \eta\rangle$ the eigenstates solutions of equation (2) in the basis where $L^{2}$ is diagonal. (Note that $k$ is the eigenvalue of $\mathcal{L}_{3}$ and not of $L_{z}$ ). Using (91) one obtains two expressions for the average of $L_{x}$ and $L_{y}$ in the particular case of a real value of $\eta$ :

$$
\begin{array}{llll}
\left\langle L_{x}\right\rangle=k \sqrt{1-\eta^{2}}, \quad\left\langle L_{y}\right\rangle=0, & \text { if } & |\eta|<1 \\
\left\langle L_{x}\right\rangle=0, \quad\left\langle L_{y}\right\rangle=k \sqrt{\eta^{2}-1}, & \text { if } & |\eta|>1 .
\end{array}
$$

The case with $|\eta|=1$ is obviously singular but there is a unique well known solution for which $w=0$. One has then

$$
\left(L_{x} \pm i L_{y}\right)|l, k= \pm l, \eta= \pm 1\rangle=0
$$

and $|l, \pm l, \eta= \pm 1\rangle$ coincide with the eigenstates of $L_{z}$ with eigenvalues $\pm l$. If $\eta$ takes a complex value $\left\langle L_{x}\right\rangle$ and $\left\langle L_{y}\right\rangle$ are both nonzero but are both proportional to $k$. The intelligent spin states are the solutions of equation (2) with average values given by (10) or (11) and which satisfy moreover equation (71). The generalized intelligent states or the quasi-intelligent spin states which were respectively defined in [1] and [3] are the solutions of equation (2) with a complex value of $\eta$ for which both averages are nonzero and to which equation (5) must be applied with a nonzero value of $\alpha$. In both cases it is sufficient to consider the interval $|\eta|<1$ and $\alpha \in[0, \pi / 2]$.

Let us now classify those states according to $k$ as follows:

i) The states with $k=0$ for which $\left\langle L_{x}\right\rangle$ and $\left\langle L_{y}\right\rangle$ are zero and only $\left\langle L_{z}\right\rangle$ is nonzero.

ii) The states with $k= \pm l$ which are very particular as we will show below.

iii) The states with intermediate values of $k$.

In order to justify this classification we have to express $\left\langle L_{z}\right\rangle$ as

$$
\left\langle L_{z}\right\rangle=\eta\left\langle\mathcal{L}_{3}\right\rangle+i \sqrt{1-\eta^{2}}\left\langle L_{y}\right\rangle-\left\langle\mathcal{L}_{+}\right\rangle .
$$

Except for the case $k= \pm l$ the average of the operator $L_{x}+i \eta L_{y}$ defined by equation (8) is nonzero. This average can be calculated from the works of [1] or [3]. It is generally the 
ratio between two polynomials of the parameter $\eta$, the degree of which increases with $l$. Therefore the angle between the vector $\langle\vec{L}\rangle$ and the $O z$ axis is not a simple function of $\eta$ for general values of $k$ and $l$ and there is no simple geometrical meaning of this angle. For $k= \pm l$ such an interpretation can indeed be found. Let us define $\eta$ in terms of two angles $\theta_{0}$ and $\phi_{0}$ by

$$
\eta=\frac{\tan \phi_{0}+i \cos \theta_{0}}{\cos \theta_{0} \tan \phi_{0}+i}
$$

With this value of $\eta$ the equation

$$
\left(L_{x}+i \eta L_{y}\right)|l, l, \eta\rangle=l \sqrt{1-\eta^{2}}|l, l, \eta\rangle
$$

can be written simply as

$$
(\vec{L} \cdot \vec{l})|l, l, \eta\rangle=l|l, l, \eta\rangle,
$$

where the vector $\vec{l}$ is the unit vector in the direction of $\langle\vec{L}\rangle$ which is such that

$$
\left\langle L_{z}\right\rangle=l \sin \theta_{0} \cos \phi_{0}, \quad\left\langle L_{y}\right\rangle=l \sin \theta_{0} \sin \phi_{0}, \quad\left\langle L_{z}\right\rangle=l \cos \theta_{0} .
$$

Equation (17) expresses the fact that $|l, l, \eta\rangle$ are simply spherical harmonics $Y_{l}^{l}$ in a system of axis where $O z$ is along the vector $l$. These states are called generally Radcliffe's states $[7]$. Let $\vec{u}, \vec{v}, \vec{l}$ be three unit vectors forming an orthogonal direct system. One has also

$$
(\vec{L} \cdot(\vec{u}+i \vec{v})|l, l, \eta\rangle=0 .
$$

In this rotated system of coordinates the parameter $\eta$ is equal to one!

For $k \neq \pm l$ the transformation which enables to build up the intelligent or quasiintelligent spin states from the usual spherical harmonics is not a rotation. This remark was already formulated long ago in [3] at the same time as the previous remark on the equivalence of Radcliffe's states with the states with $k= \pm l$.

\section{Study of a family of exponential wave packets}

Let us now discuss the properties of wave packets built from intelligent or quasiintelligent spin states with the same value of $w$, i.e. of $k$, and containing many different values of $l$. The consideration of such admixtures enables indeed to modify at our will the angular spread keeping either (5) or (7) and with the allowance, provided by equation (3), that $\eta$ is an adjustable squeezing parameter. These WP can be expressed either in the basis $|l, k, \eta\rangle$ with coefficients $c_{l}(k, \eta)$ as

$$
\left|\Psi_{\eta k}\right\rangle=\sum_{l} c_{l}(k, \eta)|l, k, \eta\rangle
$$

or in the basis of ordinary spherical harmonics with coefficients $b_{l m}(k, \eta)$ as

$$
\Psi_{\eta k}(\theta, \phi)=\sum_{l m} b_{l m}(k, \eta) Y_{m}^{l}(\theta, \phi) .
$$

I One also finds other cases where the states coincide with spherical harmonics with an axis of quantization different from $O z$ : it is the $O x$ axis for $\eta=0$ and for $\eta=i|\eta|$ this axis has $\theta=\pi / 2$ and $\sin \phi=-|\eta| / \sqrt{1-\eta^{2}}$. 
We will discuss from now on the properties of families of states generated from a parent state investigated by us elsewhere [4] and called the exponential coherent state. For a real value of $\eta$ this state depends on a single parameter $N$ and a function of $\theta$ and $\phi$ called $v$, its expression is:

$$
\Psi_{\eta 0}(\theta, \phi)=\sqrt{\frac{N}{2 \pi \sinh 2 N}} e^{N v}=\sqrt{\frac{N}{2 \pi \sinh 2 N}} e^{N \sin \theta(\cos \phi+i \eta \sin \phi)} .
$$

The probability density which is associated depends only on $N$. It is maximum within a solid angle symmetric around the axis $O x$ and the width of this solid angle is of the order $4 \pi /(4 N+1)$. Also the average $\left\langle L_{z}\right\rangle$ obeys the following formula

$$
\left\langle L_{z}\right\rangle=\eta\left(N \operatorname{coth}(2 N)-\frac{1}{2}\right) \stackrel{N \gg 1}{\longrightarrow} \eta\left(N-\frac{1}{2}\right) .
$$

We have shown in [4] how to obtain this WP from a three dimensional harmonic oscillator coherent state. As said in [4 all the WP have $k=0$. Our purpose is now to construct for real or complex $\eta$ families of WP having increasing values of $k$ using (21) for the parent state. We would like to study the angular spread as a function of $k$ and so to say the coherence properties of the following family:

$$
\Psi_{\eta 0}, \quad \mathcal{L}_{+} \Psi_{\eta 0}, \quad \mathcal{L}_{+}^{2} \Psi_{\eta 0}, \ldots, \mathcal{L}_{+}^{k} \Psi_{\eta 0}
$$

These states are all solutions of equation (2) with respectively eigenvalues $0, \sqrt{1-\eta^{2}}, 2 \sqrt{1-\eta^{2}}, \ldots, k \sqrt{1-\eta^{2}}, \ldots$ If $\eta$ is real they all satisfy equation (17) while equation (5) is satisfied if $\eta$ is complex. Applying $\mathcal{L}_{+}$onto the argument $v$ of (21) one obtains a function $v_{+}$defined by

$v_{+}=\mathcal{L}_{+} v=\frac{1}{2}(\cos \theta-\eta) \sqrt{\frac{1+\eta}{1-\eta}}-\frac{1}{2}(\cos \theta+\eta) \sqrt{\frac{1-\eta}{1+\eta}}+(\eta \cos \phi+i \sin \phi) \sin \theta$.

But a second application leads to the following property:

$$
\mathcal{L}_{+} v_{+}=\mathcal{L}_{+}^{2} v=0 \text {. }
$$

The set defined by (23) can be expressed then simply in terms of $\Psi_{\eta, 0}$ and of powers of $v_{+}$as

$$
\Psi_{\eta 0}, \quad v_{+} \Psi_{\eta 0}, \quad v_{+}^{2} \Psi_{\eta 0}, \ldots, v_{+}^{k} \Psi_{\eta 0} .
$$

In this manner one sees that the gaussian will be dominant for any $k$ in such a way that the WP will be highly concentrated on the sphere even for high $k$. The action of the operator $\mathcal{L}_{+}^{k}$ is however very different if one analyses the decomposition of WP into partial waves according to the expansion (20). Indeed it suppress all the partial waves with $l<k$, it moves the distribution towards the values with $m>l$ and also, by the change in the normalization, it increases the weight of the higher partial waves. However if one uses not the basis of the usual spherical harmonics (20) but the basis of intelligent spin states one can draw benefit from the fact that we have in this basis the equation

$$
\mathcal{L}_{+}|l, k, \eta\rangle=\sqrt{l(l+1)-k(k+1)}|l, k+1, \eta\rangle
$$


which shows that the relative phases of the states present in (19) are not affected. Therefore the states will keep their coherence property as a function of $k$.

To be complete we give below the expression of $b_{l m}(0, \eta)$ from which a recurrence can be built easily to find the coefficients for $k>0$. One has found in [4] that for real values of $\eta$

$b_{l m}=\sqrt{\frac{2 N}{\sinh (2 N)}} \sum_{l_{1} l_{2}}(-1)^{l_{2}} \frac{(N(1+\eta))^{l_{1}}(N(1-\eta))^{l_{2}}}{\sqrt{\left(2 l_{1}\right) !\left(2 l_{2}\right) !}} \frac{\left\langle l_{1} l_{2} 00 \mid l 0\right\rangle\left\langle l_{1} l_{2} l_{1}-l_{2} \mid l m\right\rangle}{\sqrt{(2 l+1)}}$.

For complex $\eta$ the square root in front must be changed.

The distribution of the coefficients $b_{l m}$ is presented in figure 1 for $\eta=0.5, N=0$ and $k=0,10$ and 20. A very large shift of the distribution of the $b$ 's towards the higher $l$ and $m>l$ is clearly observed when $k$ increases as well as an increase in the spread of the values of $m$.

Sections of the WP at their maximum are presented in figure 2 which shows the angular shape of the WP. Apart from a small movement towards smaller values of $\theta$ when $k$ increases the WP is almost as strongly localized for $k=20$ as it was for $k=0$ for $\eta=0.5$ and $\alpha=0$, as well as for $\alpha \neq 0$.

Despite the strong angular concentration of the WP for $k=20$ the calculation of $\left\langle L_{z}\right\rangle$ provides very large values for the product of the uncertainties. This average value is shown in figure 3 for $\eta=0.5$ in the same time as $\left\langle L^{2}\right\rangle$.

\section{Fractional revivals for the case of a rigid rotation}

The time evolution of the wave packets described above can now be studied assuming the hamiltonian (11). This assumption was applied in [4] for the state (21), i.e the state representing a rigid heteronuclear molecule and we have made an extensive study of its time evolution. The fractional waves that one obtains as time proceeds are obtained from the initial WP by multiplying the $b_{l m}$ by $\exp \left(-i I \omega_{0} t_{s}\right.$ ) (see [4]) where $\omega_{0}$ is the frequency of periodicity of the rigid rotor and where $t_{s}$ is a fractional time. It is this change of $t_{s}$ which allows to obtain a rich variety of fractional waves on the sphere for $k=0$. Depending on the value of $\eta$ (only real values of $\eta$ were considered in [4]) the WP exhibits a rich scenario of fractional revivals in the line described in [8], the origin of which being traced in the quantum mechanical spreading. The parameter $\eta$ allows to control the relative spread of each of the angular variables. For fractional time $(m / n) T_{\text {rev }}$, where $T_{\text {rev }}$ is a common revival time, the WP is subdivided into a certain number of WP ( $n$ if $n$ is odd, $n / 2$ in the even case) the shape of which depend strongly on the value of $\eta$. For the case $\eta= \pm 1$ the fractional WP are clones of the initial WP. For different values their shape change (we have called these WP mutants). If $N$ is large enough the fractional WP are located around different directions on the sphere and do not interfere much spatially for low enough values of $n(n<8$ for $N=20)$. We interpret this properties as a manifestation of a robust virtue of coherence of the WP. The revivals of various WP having $k=0,5,10$ and 20 are presented for $m / n=1 / 10$, 
such a time has been chosen as a typical exemple. There we should observe 5 wave packets according to [8]. These WP are always very well separated from each other, again there is no significant difference between the WPs constructed from intelligent spin states and those built from quasi-intelligent ones.

\section{Conclusions}

The conclusion of this article is that there exists very numerous possibilities of construction of angular coherent states using the properties of the intelligent spin states. For a system with an hamiltonian quadratic in $I[I(I+1)$ spectrum $]$ these WP spread on the sphere but there is a well identified mechanism of fractional revivals that produce a set of well concentrated mutants. For rotators which are not quadratic the scenario is valid during a limited time. This is the case of nuclei for which we are making a parallel study [9].

\section{Acknowledgment}

One of us (P.R.) kindly acknowledge support of Polish Committee for Scientific Research (KBN) under the grant 2 P03B 14314.

\section{References}

[1] Aragone C, Guerri G, Salamo S and Tani JL 1974 J. Phys. A: Math. Nucl. Gen. 7 L149 Aragone C, Chalbaud E and Salamo S 1976 J. Math. Phys. 171963

[2] Kolodziejczyk L and Ryter A 1974 J. Phys. A: Math. Nucl. Gen. 7213 Vetri G 1975 J. Phys. A: Math. Nucl. Gen. 8 L55 Kolodziejczyk L 1975 J. Phys. A 8 L99

[3] Rashid M A 1978 J. Math. Phys. 191391 Rashid M A 1978 J. Math. Phys. 191397

[4] Rozmej P and Arvieu R 1998 Phys. Rev. A 584314

[5] Jackiw R 1968 J. Math. Phys. 9339

[6] Levy-Leblond J M 1986 Am. J. Phys. 54135

[7] Radcliffe J M 1971 J. Phys. A 4313

[8] Averbukh I Sh and Perelman N F 1989 Phys. Lett. A 139449

[9] Rozmej P and Arvieu R 1999 to be published in Acta Phys. Pol. B (preprint quant-ph/9809073) 


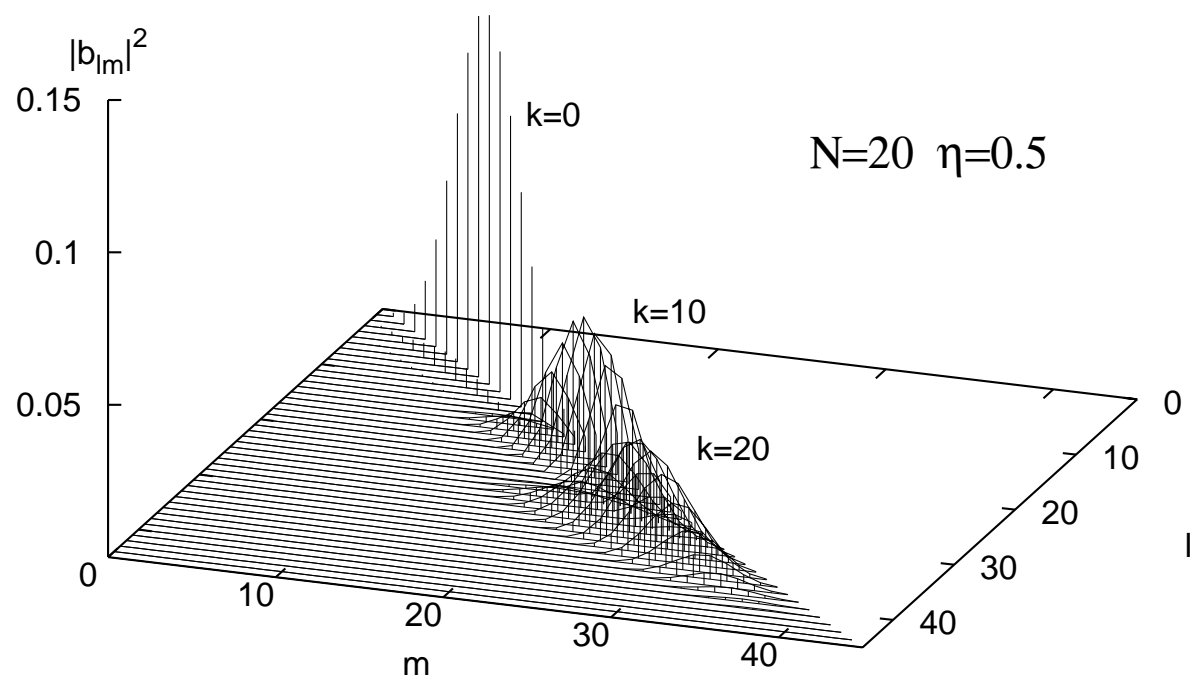

Figure 1. Distribution of weights $\left|b_{l m}\right|^{2}$ for WP with $N=20, \eta=0.5$ and three values of $k$. The cases with $k=10$ and 20 are plotted both in lines and impulses. 


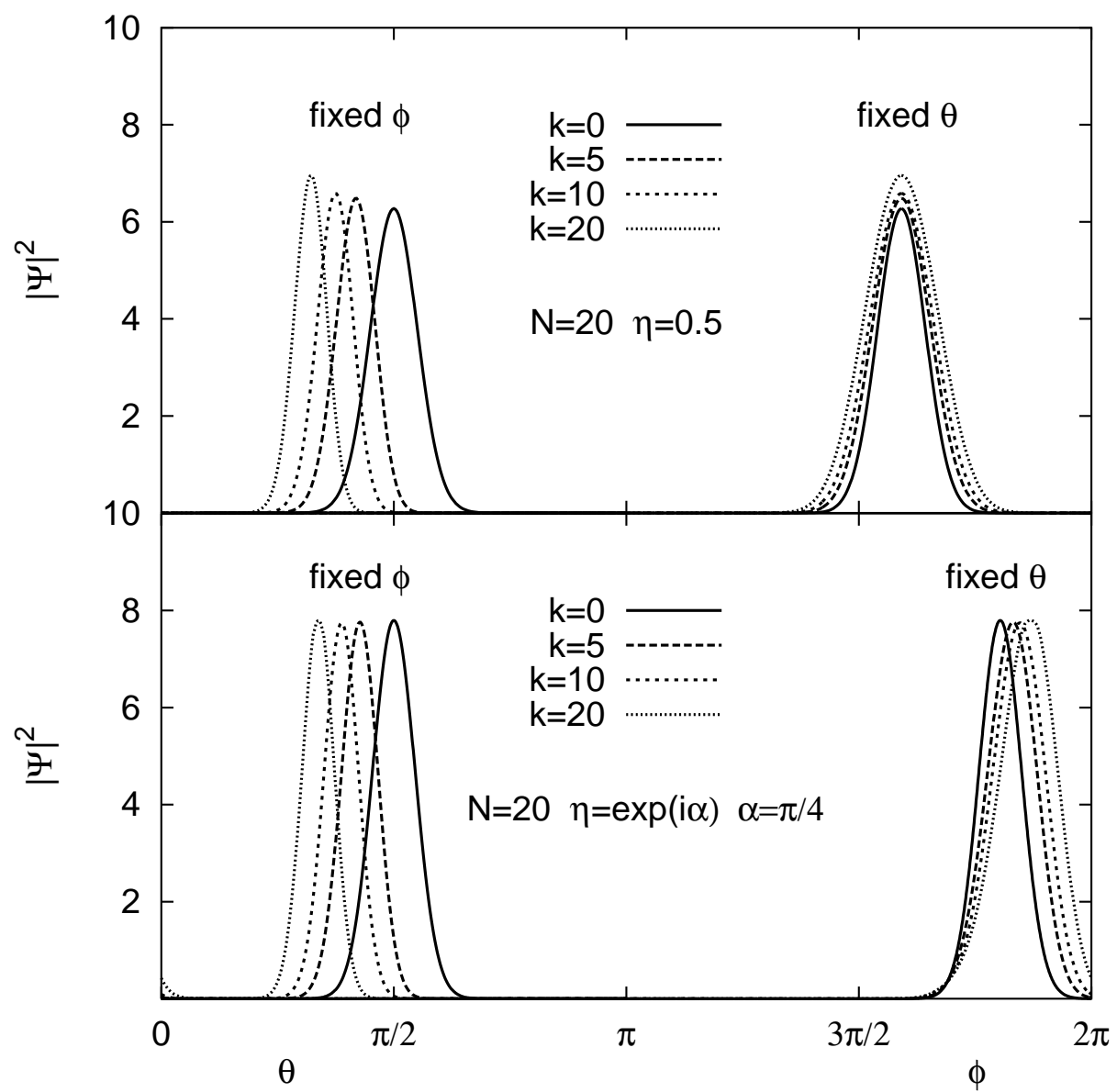

Figure 2. Sections, in $\theta$ and $\phi$ directions, through the WP's maxima at time $t=0$ for WP with $N=20, \eta=0.5$ (top), and those with $N=20, \eta=\exp (i \alpha), \alpha=\pi / 4$ (bottom) and four $k$ values. In top part all sections in $\phi$ are shifted from $\phi=0$ to $\phi=5$ for more convenient presentation. 


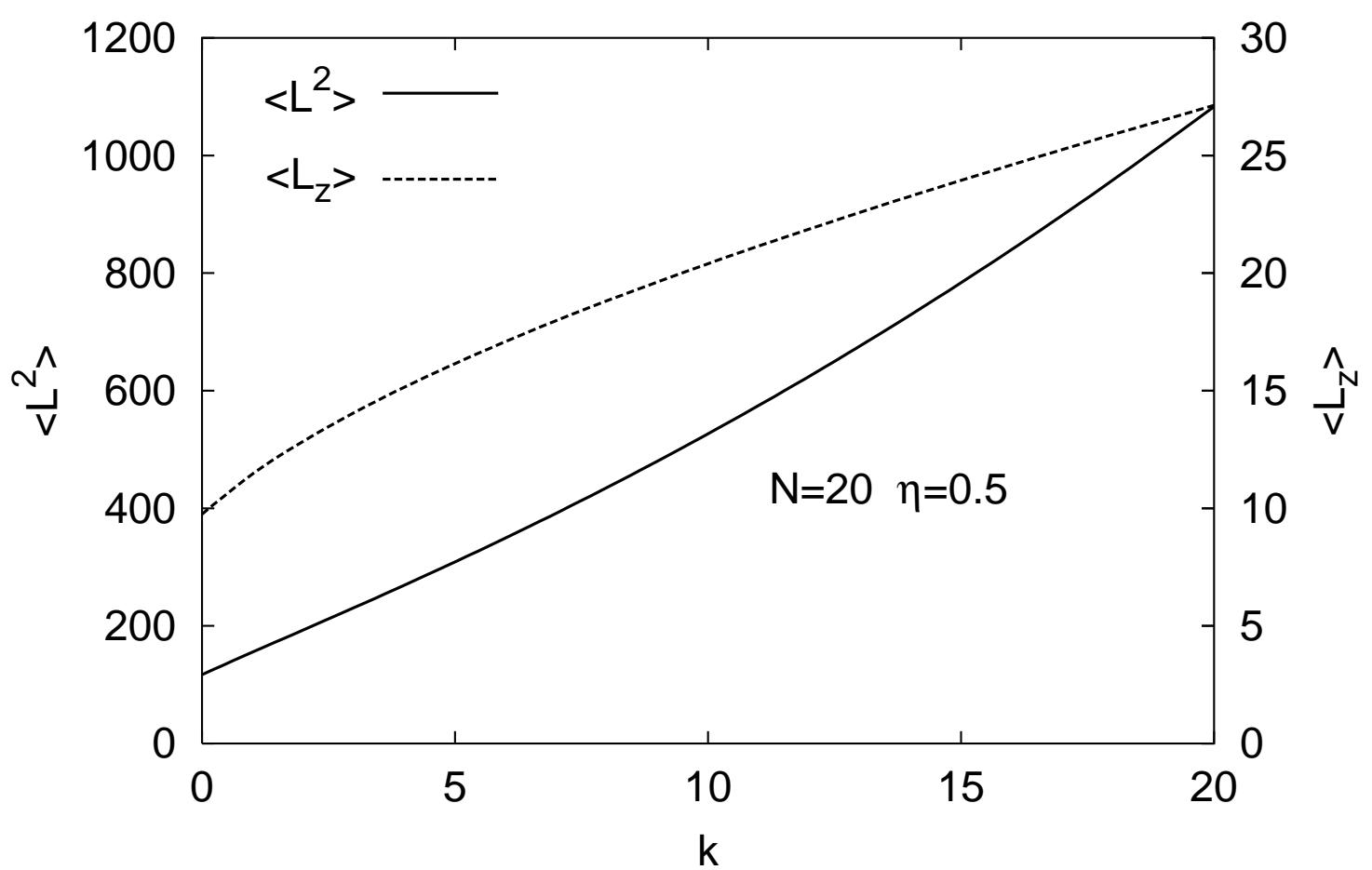

Figure 3. Expectation values of $L^{2}$ (left scale) and $L_{z}$ (right scale) operators for WP with $N=20, \eta=0.5$ as functions of $k$. 

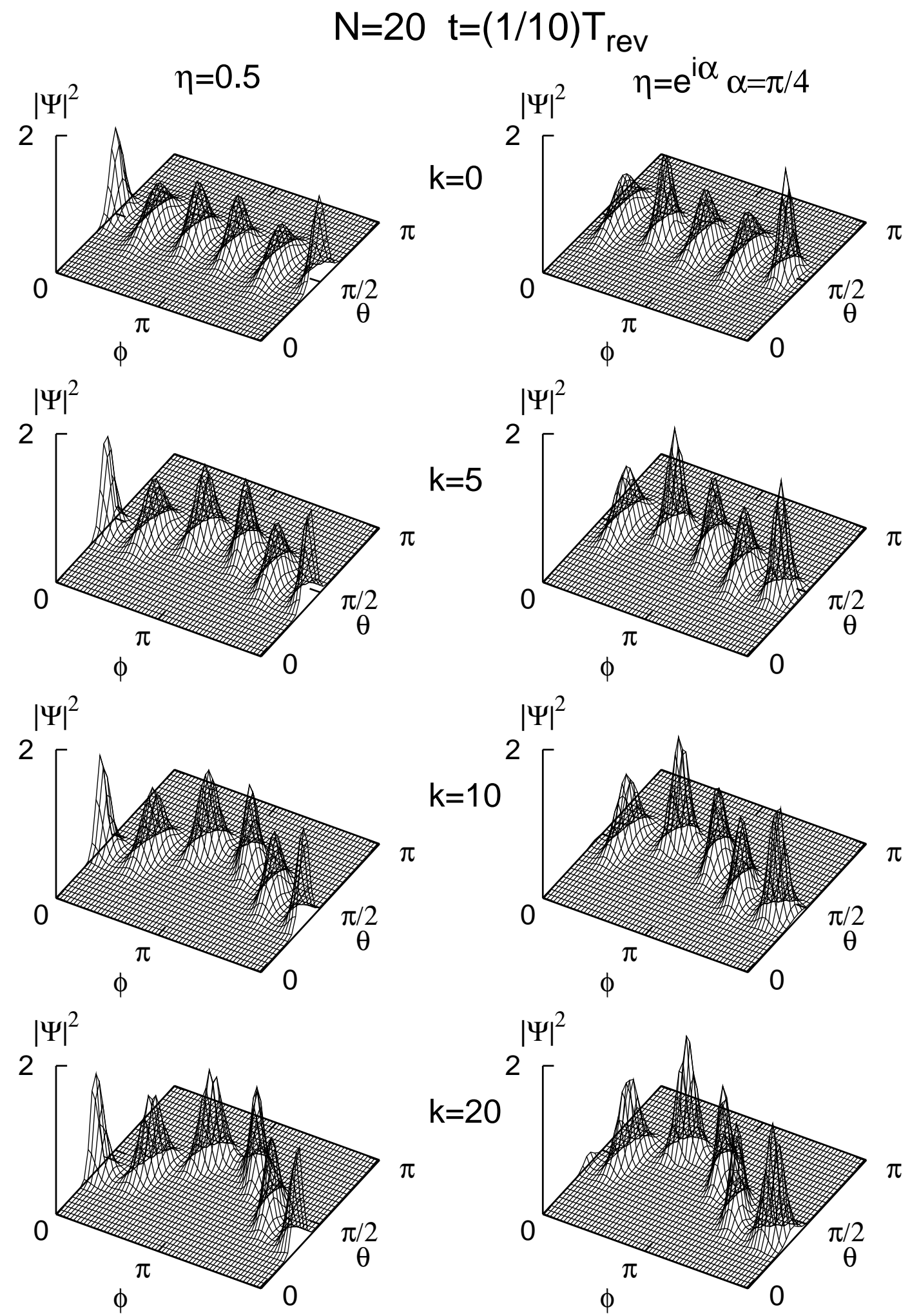

Figure 4. Shapes of wave packets with $N=20$ at fractional revival time $t=$ $(1 / 10) T_{\text {rev }}$ for real $\eta=0.5$ (left column), and $\eta=\exp (i \alpha), \alpha=\pi / 4$ (right column) and $k=0,5,10,20$ as funtions of angular variables for a rigid molecule. Clones and mutants are clearly visible. Note that with increasing $k$ the classical trajectory becomes more and more tilted with respect to $O x y$ plane. 